\title{
Burstiness and Aging in Social Temporal Networks
}

\author{
Antoine Moinet, Michele Starnini, ${ }^{*}$ and Romualdo Pastor-Satorras \\ Departament de Física i Enginyeria Nuclear, Universitat Politècnica de Catalunya, Campus Nord B4, 08034 Barcelona, Spain
}

(Received 26 November 2014; published 13 March 2015)

\begin{abstract}
The presence of burstiness in temporal social networks, revealed by a power-law form of the waiting time distribution of consecutive interactions, is expected to produce aging effects in the corresponding time-integrated network. Here, we propose an analytically tractable model, in which interactions among the agents are ruled by a renewal process, that is able to reproduce this aging behavior. We develop an analytic solution for the topological properties of the integrated network produced by the model, finding that the time translation invariance of the degree distribution is broken. We validate our predictions against numerical simulations, and we check for the presence of aging effects in a empirical temporal network, ruled by bursty social interactions.
\end{abstract}

DOI: 10.1103/PhysRevLett.114.108701

PACS numbers: $89.75 . \mathrm{Hc}$

Our understanding of the structure and properties of social interactions has experienced a boost in recent years due to the new availability of large amounts of digital empirical data [1,2]. This endeavor has found the necessary theoretical grounding in network science [3,4]. A first round of studies focused on a static network representation $[5,6]$, in which nodes (standing for individuals) and edges (indicating social interactions) are constant and never change in time. From such a static representation, a wealth of complex topological information was obtained, concerning, e.g., the presence of scale-free, power-law degree distributions $P(k) \sim k^{-\gamma}$, large clustering, positive degree correlations, or a distinct community structure [7]. More recently, this framework has been challenged by the empirical observation of a temporal dimension in networks, particularly evident in social systems, due to the fact that social relationships are continuously created and terminated. From these temporal networks [8], a static representation is obtained by means of an integration of the instantaneous interactions over a time window of width $t$, and its associated topological properties, such as the degree distribution $P_{t}(k)$, are thus to be understood to depend on the integration time [9]. The empirical study of the temporal aspects of social networks has unveiled an additional level of complexity, embodied in many statistical properties showing heavy-tailed distributions. Remarkable among them are the distribution $\psi(\tau)$ of interevent or waiting times between two consecutive social interactions, revealing the bursty nature of human dynamics, and the distribution $F(a)$ of social activity, measuring the probability per unit time of establishing a new social relation. Both distributions approximately obey power-law decays of the form $\psi(\tau) \sim \tau^{-(1+\alpha)}$ and $F(a) \sim a^{-\delta}$, respectively [10-14]. Noteworthy, the bursty nature of social interactions represents a common feature of human dynamics that can be related to the prioritized behavior of human beings [11].
This twofold nature of social interactions naturally raises the issue of the relation between the temporal correlation properties of time-varying networks and the topological features of their static representations. Among others, Song et al. [15] proposed an empirical scaling theory bridging the exponents of human dynamics patterns and social network architecture, while Perra et al. [14] considered an activity driven model, built upon the idea of a constant social activity, defined as the probability per unit time that an agent becomes active and starts a social interaction. The activity driven model allows us to show that the degree distribution $P_{t}(k)$ of an integrated network is functionally related to the distribution of social activity $F(a)$ by $P_{t}(k) \sim$ $t^{-1} F[(k / t)-\langle a\rangle][16]$. Following this direction, in this Letter we focus on a different property of social temporal networks, naturally expected in systems in which the addition of connections is ruled by a non-Poissonian, power-law distribution of interevent times, namely, the presence of aging behavior $[17,18]$. Indeed, the construction of integrated networks assumes implicitly an integration interval $[0, t]$. One can however consider a more general time interval $\left[t_{a}, t_{a}+t\right]$, spanning a width $t$, after an aging time $t_{a}$. While the effects of a varying integration window have been already considered in the literature of temporal networks $[9,19]$, here we focus on an aging behavior translating into a breaking of time translation invariance, manifested in the dependence of the topological properties of the integrated network on the time $t_{a}$ at which the integration starts. We notice that this notion of aging is closely related to the aging observed in renewal theory $[20,21]$, which is not due to the physical aging of agents, i.e., to the possible changes of the activity or connectivity patterns of agents because of their becoming old, but it is uniquely due to the heavy-tailed form of their interevent time distribution. Physical aging phenomenology has been considered in standard nontemporal networks in Refs. [22,23] (see also Ref. [24]). 
In order to study this possibility from an analytic point of view, we propose and analyze a non-Poissonian activity driven (NoPAD) model, in which the waiting time between consecutive agent activations follows an arbitrary form $\psi_{i}\left(\tau, c_{i}\right), c$ being a parameter quantifying the (possible) heterogeneity of waiting times in the population. We compute the topological properties of the ensuing integrated networks by applying the hidden variables formalism $[16,25]$. We find that if $\psi(\tau, c)$ is a power-law distribution with exponent $1+\alpha$, then the degree distribution exponent $\gamma$ is simply related to $\alpha$, mediated by the heterogeneity distribution $\eta(c)$. In this model, effects of aging are clearly evident. We observe in particular that both the degree distribution $P_{t_{a}, t}(k)$ and the average degree $\langle k\rangle_{t_{a}, t}$, computed in a time window $\left[t_{a}, t_{a}+t\right]$, depend explicitly on the initial integration time $t_{a}$. Evidence of this sort of aging is recovered in an empirical analysis of the temporal network defined by the scientific collaborations in the journal Physical Review Letters (PRL), published by the American Physical Society (APS) [26].

Previous modeling efforts have shown that the concept of memory can induce non-Poissonian interevent time distributions in temporal networks [27]. Here, we propose a model joining the activity driven framework with the empirically observed bursty nature of social interactions, which allows for a simple mathematical treatment. The NoPAD model is defined as follows. Each agent $i$ in a network of size $N$ is endowed with a time-dependent activity $a_{i}(t)$, which represents the probability per unit time that agent $i$ becomes active for the first time after a time $t$ from its last activation. When an agent becomes active, it generates an edge that is connected to another agent chosen uniformly at random. Edges last for a period of time that we assume to be infinitesimally small. The activation of each individual follows thus a renewal process [20] with an interevent time distribution given by [20] $\psi_{i}(\tau)=a_{i}(\tau) \exp \left\{-\int_{0}^{\tau} a_{i}\left(\tau^{\prime}\right) d \tau^{\prime}\right\}$. (We assume that the time between activation events is not affected by the reception of a connection emitted by other active agents.) For a time-independent activity $a_{i}$ we recover the original activity driven model [14], with a Poissonian, exponential interevent time distribution. An explicitly time-dependent activity rate leads to a non-Poissonian activity pattern, which can take the power-law, bursty form found in human interactions. Shifting from the activity (failure rate) $a_{i}(t)$ to the equivalent waiting time distribution $\psi(\tau)$, we define the NoPAD model in a operational way. Each agent in the network becomes active following a renewal process ruled by a waiting time distribution $\psi\left(\tau, c_{i}\right)$, where $c$ is a parameter gauging the heterogeneity of the activation rate of the agents, which we assume to be randomly distributed according to a distribution $\eta(c)$. Active agents connect to a randomly chosen node by an edge that lasts an infinitesimally small time.
The topological properties of the integrated networks emerging from the NoPAD model can be computed by applying the general hidden variables formalism [16,25]. Hidden variables network models consider a set of $N$ nodes, each one of them being assigned a hidden variable $\vec{h}$, drawn from a probability distribution $\rho(\vec{h})$. For each pair of vertices $i$ and $j, i \neq j$, an edge is created with connection probability $\Pi\left(\vec{h}_{i}, \vec{h}_{j}\right)$. The resulting network has the degree distribution

$$
P(k)=\sum_{\vec{h}} \rho(\vec{h}) g(k \mid \vec{h}),
$$

where the propagator $g(k \mid \vec{h})$ is the conditional probability that a vertex with hidden variable $\vec{h}$ ends up with degree $k$, and whose generating function $\hat{g}(z \mid \vec{h})=\sum_{k} z^{k} g(k \mid \vec{h})$ fulfills the equation [25]

$$
\ln \hat{g}(z \mid \vec{h})=N \sum_{\overrightarrow{h^{\prime}}} \rho\left(\vec{h}^{\prime}\right) \ln \left[1-(1-z) \Pi\left(\vec{h}, \vec{h}^{\prime}\right)\right] .
$$

Focusing for simplicity in an integration interval $[0, t]$, and assuming that all agents are synchronized at time $t=0$, the key point to map the NoPAD model to a hidden-variable model resides in computing the probability $\Pi_{t}(i, j)$ that two vertices $i$ and $j$ become eventually linked by time $t$. Following Ref. [16], this probability can be written as $\Pi_{t}(i, j) \equiv \Pi_{t}\left(r_{i}, r_{j}\right)=1-[1-(1 / N)]^{r_{i}}[1-(1 / N)]^{r_{j}}$, where $r_{i}$ is the number of times node $i$ has become active up to time $t$. This number of activations is itself a random variable with distribution $\chi_{t}(r \mid c)$, depending on the agent's heterogeneity $c$ and time $t$ [20]. The mapping to a hidden variables network is now clear: the hidden variables are the vector $\vec{h} \rightarrow(r, c)$, with a probability distribution $\rho(\vec{h}) \rightarrow \rho_{t}(r, c) \equiv \eta(c) \chi_{t}(r \mid c)$, and the connection probability takes the form $\Pi\left(\vec{h}, \vec{h}^{\prime}\right) \rightarrow \Pi_{t}\left(r, r^{\prime}\right) \simeq\left(r+r^{\prime}\right) / N$, independent of $c$ and $c^{\prime}$, in the limit $N \gg 1$ and $N \gg r, r^{\prime}$. Applying this mapping to Eq. (2) we obtain $\hat{g}(z \mid r, c) \simeq \exp \left[(z-1)\left(\langle r\rangle_{t}+r\right)\right]$. The resulting propagator [28] is a Poisson distribution, sharply peaked at its average value $r+\langle r\rangle_{t}$, where $\langle r\rangle_{t}=\sum_{c} \eta(c) \sum_{r} r \chi_{t}(r \mid c)$ is the average number of activation events at time $t$. Applying a steepest descent approach [16] leads, through Eq. (1), to an approximate expression for the degree distribution of the integrated network in the interval $[0, t]$

$$
P_{t}(k) \simeq \sum_{c} \eta(c) \chi_{t}\left(k-\langle r\rangle_{t} \mid c\right) .
$$

The remaining element to close the calculation is the probability $\chi_{t}(r \mid c)$, whose expression can be easily worked out in Laplace space [20]. For the empirically relevant case of heavy-tailed waiting time distributions with form

$$
\psi(t, c)=\alpha c(c t+1)^{-(\alpha+1)}, \quad 0<\alpha<1,
$$


corresponding to a time-dependent activity $a(t, c)=$ $\alpha c /(1+c t)$, we can use the approximation developed in Ref. [29], valid for large $r /(c t)^{\alpha}$, namely,

$$
\chi_{t}(r \mid c) \sim \frac{1}{(c t)^{\alpha}} e^{\xi(\alpha, c t) r^{1 /(1-\alpha)}},
$$

where $\xi(\alpha, u)=-[1-\alpha]\left[(\alpha / u)^{\alpha} \Gamma(1-\alpha)\right]^{1 /(1-\alpha)}$. From Eq. (3), the degree distribution of the integrated network up to time $t$ is given, in the continuous $c$ limit, by

$$
P_{t}(k) \sim \frac{\left(k-\langle r\rangle_{t}\right)^{(1-\alpha) / \alpha}}{t} \int d u \eta\left(\frac{u}{t}\left[k-\langle r\rangle_{t}\right]^{(1 / \alpha)}\right) \frac{e^{\xi(\alpha, u)}}{u^{\alpha}} .
$$

As we will argue below, a reasonable form for the heterogeneity distribution is a power-law one,

$$
\eta(c)=\frac{\beta}{c_{0}}\left(c / c_{0}\right)^{-(\beta+1)}
$$

with $\beta>\alpha$. The degree distribution is thus given, for $k \gg\left(c_{0} t\right)^{\alpha}$, by

$$
P_{t}(k) \sim\left(c_{0} t\right)^{\beta}\left(k-\langle r\rangle_{t}\right)^{-1-(\beta / \alpha)} .
$$

Equation (7) establishes the relation between the exponent of the power-law degree distribution $P(k) \sim k^{-\gamma}$ and the exponent of the long-tailed waiting time distribution $\psi(t) \sim t^{-1-\alpha}$, namely,

$$
\gamma=1+\beta / \alpha .
$$

This relation, mediated through the exponent $\beta$, manifests the relevance of the assumed distribution of heterogeneity $\eta(c)$. We can motivate the form assumed for this distribution by relating it to the empirical activity measurements performed in Ref. [14]. There, the average activity of an individual $i$ over a time interval $\bar{a}_{i}(\Delta t)$, defined as the ratio between the number of social acts performed by individual $i$ in the time interval $\Delta t$, and the total number of social acts in the system in that interval, was actually measured. In the NoPAD model, the number of social acts of an individual with heterogeneity $c$ in an interval $\Delta t$ is determined by the number of times he has become active in that interval, which from Eq. (9) (see below) is given by $\bar{r}_{\Delta t}(c) \sim c^{\alpha}(\Delta t)^{\alpha}$. Therefore, we have $\bar{a}(c) \sim c^{\alpha}$, independent of $\Delta t$. A simple transformation between probability distributions allows us to write $\eta(c) \sim F[\bar{a}(c)][d \bar{a}(c) / d c] \sim$ $c^{-1-\alpha(\delta-1)}$. From here, we recover the postulated heterogeneity distribution (6), with an exponent $\beta=\alpha(\delta-1)$. Most remarkably, for this value of $\beta$, the integrated network shows a degree exponent $\gamma=1+\beta / \alpha=\delta$; i.e., we recover the main result of the activity driven model, stating the equivalence between degree and activity distributions [14].
In order to check our analytic predictions, we have performed numerical simulations of the NoPAD model with the waiting time and heterogeneity distributions (4) and (6), respectively. The integrated network at time $t$ is generated as follows. To each node $i$ a heterogeneity $c_{i}$ extracted from the distribution $\eta(c)$ is assigned. Then, we generate the number $r_{i}$ of times that each node becomes active up to time $t$, according to the distribution (4). Finally, each node $i$ is connected to $r_{i}$ neighbors chosen at random, avoiding multiple and self connections. In Fig. 1 we show the degree distribution $P_{t}(k)$ for different values of the exponents $\alpha$ and $\beta$ of the waiting time and heterogeneity distributions. As one can see, the scaling relation of Eq. (8) is fulfilled remarkably well. In the same figure we validate the scaling of the $P_{t}(k)$ with the integration time $t$, Eq. (7), showing the collapse of the degree distribution for different $t$.

The dependence of the topological properties of the NoPAD model on the distribution of renewal events $\chi(r \mid c)$ readily suggests that the model will be affected by aging effects when the waiting time distributions have the power-
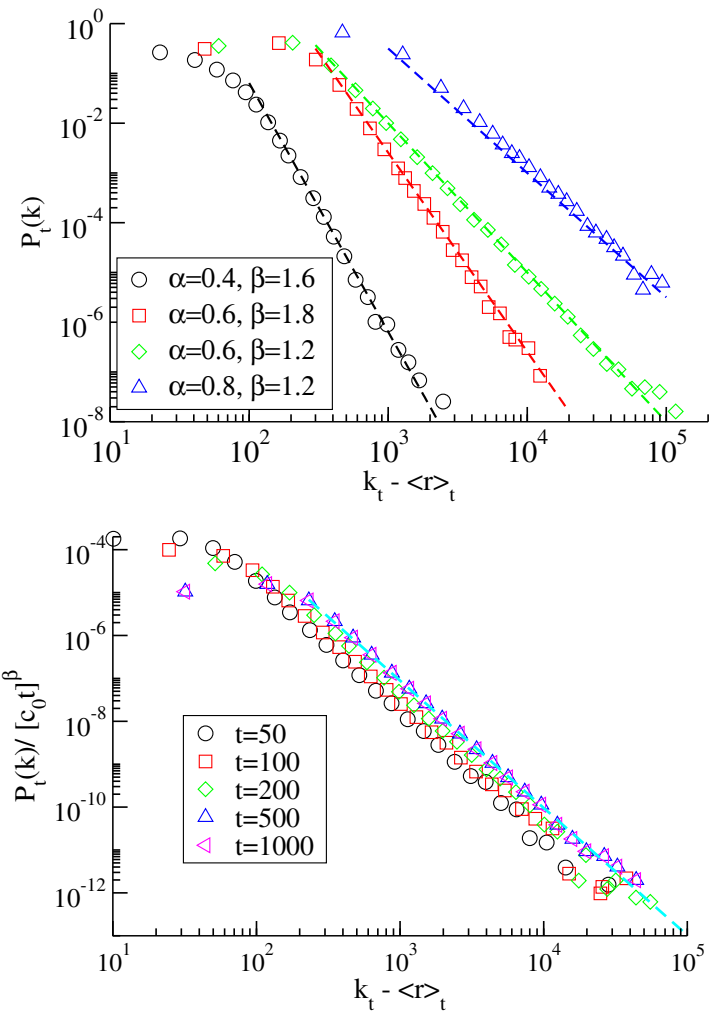

FIG. 1 (color online). Top: degree distribution $P_{t}(k)$ at time $t=10^{4}$ as a function of the rescaled degree $k-\langle r\rangle_{t}$ from numerical simulation of the NoPAD model with a network of size $N=10^{6}, c_{0}=1$, and different values of $\alpha$ and $\beta$. The exponent $\gamma$, as given by Eq. (8), is plotted as a dashed line. Bottom: rescaled degree distribution $P_{t}(k) /\left(c_{0} t\right)^{\beta}$ for different times $t$, in network size $N=10^{6}$, and $c_{0}=1, \alpha=0.6$, and $\beta=1.2$. The theoretical decay exponent $\gamma=3$ is plotted as a dashed line. 
law form (4) with $\alpha<1$ [18]. We check these effects in Fig. 2 (inset), where we plot the aged degree distribution $P_{t_{a}, t}(k)$ obtained from networks integrated for a time interval $t$, started after waiting for an aging time $t_{a}$. This figure shows that while the asymptotic shape of $P_{t_{a}, t}(k)$ remains constant for large $k$, its peak shifts to smaller values of $k$ with increasing $t_{a}$. An analytical treatment of these aging effects is in principle possible, using the results reported in Ref. [21]. We can however easily understand them at the level of the aged average degree $\langle k\rangle_{t_{a}, t}$. Since the average degree is 2 times the number of activation events, if we consider a network integrated from time $t_{a}$ to $t$ we have $\langle k\rangle_{t_{a}, t}=2\left(\langle r\rangle_{t_{a}+t}-\langle r\rangle_{t}\right)$. The average number of activation events reads, for large $t$ [29],

$$
\langle r\rangle_{t}=\sum_{c} \eta(c) \sum_{r} r \chi_{t}(r \mid c) \simeq \frac{\beta \sin (\pi \alpha)}{(\beta-\alpha) \pi \alpha}\left(c_{0} t\right)^{\alpha} .
$$

The sublinear growth of $\langle r\rangle_{t}$ readily implies aging effects on the aged average degree, translated into a nontrivial dependence of the average degree on the initial integration time $t_{a}$, which only cancels out in the case $\alpha=1$. Indeed, by applying Eq. (9) we obtain

$$
\langle k\rangle_{t, t_{a}} \sim\left[\left(t_{a}+t\right)^{\alpha}-t_{a}^{\alpha}\right] .
$$

Thus, for $t \gg t_{a}$, the average degree is independent of $t_{a}$, $\langle k\rangle_{t, t_{a}} \sim t^{\alpha} \sim\langle k\rangle_{t}$, and aging effects are negligible. On the other hand, for $t \ll t_{a}$, the average degree decays with $t_{a}$ as $\langle k\rangle_{t, t_{a}} \sim t_{a}^{\alpha-1}$, and aging effects induce an anomalous $t_{a}$ dependence, ruled by $\alpha$ [18]. These strong aging effects can be easily understood as due to an intervent time distribution lacking a first moment, i.e., with $\alpha<1$. Such a distribution implies that very large gaps between two interactions are relatively frequent. Starting the observation of the system at a random time $t_{a}$ results in a increased probability of having an integration window overlapping such frequent large gaps, in which one or more individuals do not interact. This facts immediately implies a decrease of the average degree for $t \ll t_{a}$, as analytically recovered in Eq. (10). In Fig. 2 we check that Eq. (10) correctly reproduces the behavior of the NoPAD model. In particular, we emphasize in this plot the scaling behavior, evident from Eq. (10), $\langle k\rangle_{t, t_{a}} \equiv$ $t_{a}^{\alpha} \mathcal{F}\left(t_{a} t^{-1}\right)$, with a general scaling function $\mathcal{F}(x)^{\alpha}=$ $(1-x)^{\alpha}-1$.

We explore the possibility of aging effects in empirical temporal networks by considering the scientific collaboration network in PRL, published since 1958 [26]. In this network, two authors are connected by a link if they coauthored a Letter published in PRL. Since PRL is weekly edited, time is measured in units of weeks. In order to avoid spurious effects due to effective physical aging of the population considered (i.e., scientists changing their publication rate as they become older), which are not considered in the NoPAD model, and single out the role of the

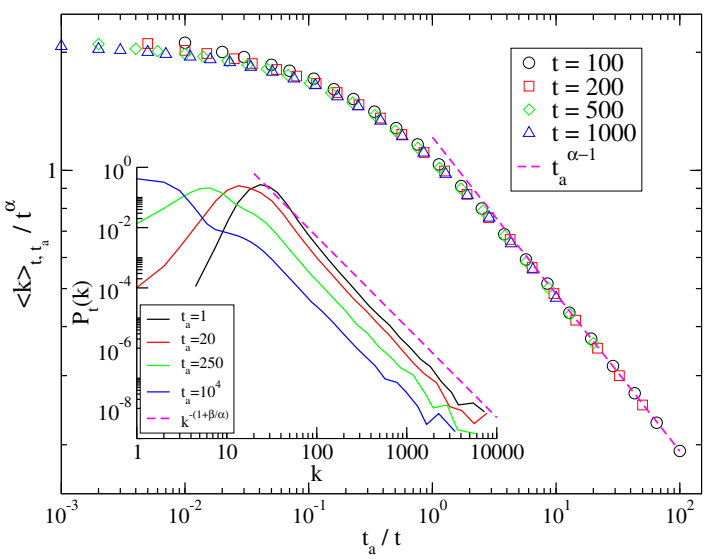

FIG. 2 (color online). Rescaled average degree $\langle k\rangle_{t_{a}, t} / t^{\alpha}$ as a function of the aging time $t_{a}$, obtained from numerical simulations of the NoPAD model with a network of size $N=10^{6}$, and parameters $\alpha=0.6, c_{0}=1, \beta=1.2$, integrated for different time intervals $t$. The behavior predicted for $t \ll t_{a}$ is shown as a dashed line. Inset: degree distribution $P_{t_{a}, t}(k)$ of the NoPAD networks of the main plot, for integration time $t=100$ and different aging time $t_{a}$. The behavior predicted by Eq. (8) is shown as a dashed line.

heavy-tailed waiting distribution, we select only those authors who published at least one paper in any APS journal before and after an interval of 30 years, spanning from 1968 up to 1998 . We then reconstruct the temporal network of the $N=677$ resulting authors, by considering those papers cowritten by two authors in this interval, and drawing an instantaneous edge between the authors at the date of the paper's publication. In Fig. 3 we check that the waiting time distribution between two consecutive publications of the same author has a clear heavy-tailed form, approximately compatible with a power-law decay

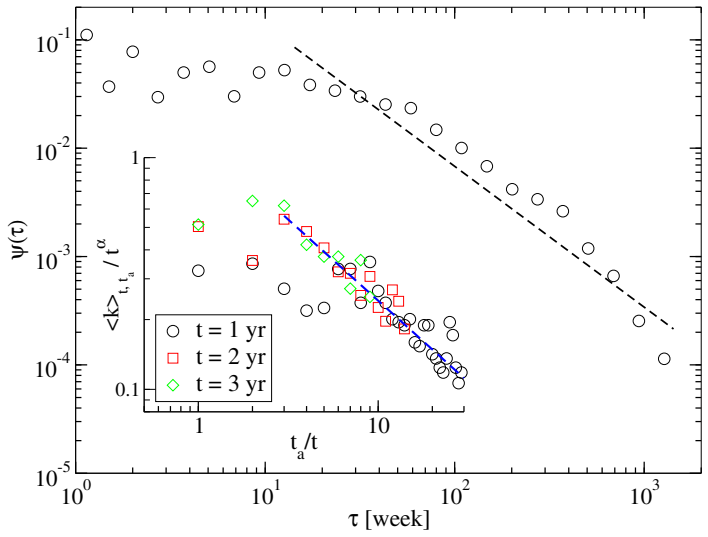

FIG. 3 (color online). Waiting time distribution $\psi(\tau)$ for two consecutive publications in PRL by the same author, in unit of weeks. A decay of the form given by Eq. (6), with $\alpha=0.3$, is plotted as a dashed line. Inset: rescaled average degree $\langle k\rangle_{t_{a}, t} / t^{\alpha}$ as a function of the aging time $t_{a}$ of the PRL temporal network, integrated for different time intervals $t=1,2$, or $3 \mathrm{yr}$. The behavior predicted by the NoPAD model for $t \ll t_{a},\langle k\rangle_{t, t_{a}} \sim t_{a}^{\alpha-1}$, is shown as a dashed line. 
$\psi(\tau) \sim \tau^{-1-\alpha}$, with exponent $\alpha \simeq 0.3$. We then proceed to construct the integrated networks, varying the aging time $t_{a}$ between 0 and 30 years and fixing the integration time as $t=1,2$, and $3 \mathrm{yr}$. In this construction it is important to realize that the actual aging time of the network is in principle unknown. Each author $i$, indeed, starts his academic life at some time $T_{i}^{0}$, included in the observational time window between 1958 and 1968. Aging effects are thus observed in networks integrated over a time window explicitly dependent on $T_{i}^{0}$ of each author considered. This point makes it extremely difficult to detect aging effects in the degree distribution $P_{t_{a}, t}(k)$, also because the low activation ratio $a_{i}(t)$ yields a very sparse network, with small degree values. Nevertheless, we are able to observe aging behavior in the average degree $\langle k\rangle_{t_{a}, t}=\sum_{k} k P_{t_{a}, t}(k)$. Figure 3 (inset) shows the aged average degree $\langle k\rangle_{t_{a}, t}$ of the empirical data, plotted in the rescaled form suggested by the NoPAD model (10). As one can see, the data are compatible with the theoretical prediction, particularly in the limit of large $t_{a}$, where we expect the actual aging time $T_{i}^{0}$ to become small with respect to $t_{a}$.

To sum up, in this Letter we addressed the aging effects observed in time-integrated networks produced by bursty social interactions. We proposed a mathematically tractable model, the NoPAD model, aimed at combining the nonPoissonian form of the waiting time distribution with the activity-driven framework, and we developed an analytic solution for its topological properties. In this analysis, we have focused on the properties of the corresponding binary integrated network, neglecting the possibility of edge weights given by the total number of connections between two nodes in the considered integration window. The formalism developed can be easily extended to deal with the statistical patterns of such a weight distribution. Aging effects are demonstrated by the dependence of the degree distribution $P_{t_{a}, t}(k)$ not only on the integration time window $t$, but also on the aging time $t_{a}$ at which we start the integration. Inspired by the results obtained in the model, we checked that aging behavior can also be observed in real temporal networks. In this respect, it is important to notice that, in real systems, the effects purely derived by a heavy-tailed interevent time distribution can be mixed with, and masked by, other features, such as finite-size effects, population fluctuations, actual aging of the individuals, memory effects, clustering, or community partitioning. The elucidation of the contribution of all these effects in the physical aging of temporal networks remains an open issue, deserving further empirical and theoretical effort.

We acknowledge financial support from the Spanish MINECO, under Projects No. FIS2010-21781-C02-01 and No. FIS2013-47282-C2-2, and the EC FET-Proactive Project MULTIPLEX (Grant No. 317532). R. P.-S. received additional financial support from ICREA Academia, funded by the Generalitat de Catalunya.
* Corresponding author. michele.starnini@gmail.com

[1] D. Lazer, A. S. Pentland, L. Adamic, S. Aral, A. L. Barabasi, D. Brewer, N. Christakis, N. Contractor, J. Fowler, M. Gutmann et al., Science 323, 721 (2009).

[2] A. Pentland, Social Physics: How Ideas Turn Into Action (Penguin Group, New York, 2014).

[3] R. Albert and A.-L. Barabási, Rev. Mod. Phys. 74, 47 (2002).

[4] M. E. J. Newman, Networks: An Introduction (Oxford University Press, Oxford, 2010).

[5] S. Wasserman and K. Faust, Social Network Analysis: Methods and Applications (Cambridge University Press, Cambridge, England, 1994).

[6] M. Jackson, Social and Economic Networks (Princeton University Press, Princeton, 2010).

[7] S. N. Dorogovtsev, Lectures on Complex Networks, Oxford Master Series in Physics (Oxford University Press, Oxford, 2010).

[8] P. Holme and J. Saramäki, Phys. Rep. 519, 97 (2012).

[9] B. Ribeiro, N. Perra, and A. Baronchelli, Sci. Rep. 3, 3006 (2013).

[10] J. G. Oliveira and A.-L. Barabasi, Nature (London) 437, 1251 (2005).

[11] A.-L. Barabasi, Nature (London) 435, 207 (2005).

[12] M. C. Gonzalez, C. A. Hidalgo, and A.-L. Barabasi, Nature (London) 453, 779 (2008).

[13] C. Cattuto, W. Van den Broeck, A. Barrat, V. Colizza, J.-F. Pinton, and A. Vespignani, PLoS One 5, e11596 (2010).

[14] N. Perra, B. Gonçalves, R. Pastor-Satorras, and A. Vespignani, Sci. Rep. 2, 469 (2012).

[15] C. Song, D. Wang, and A.-L. Barabasi, "Connections between human dynamics and network science," arXiv:1209.1411v2.

[16] M. Starnini and R. Pastor-Satorras, Phys. Rev. E 87, 062807 (2013).

[17] M. Henkel and M. Pleimling, Nonequilibrium Phase Transitions: Ageing and Dynamical Scaling far from Equilibrium (Springer Verlag, Netherlands, 2010).

[18] J. Klafter and I. Sokolov, First Steps in Random Walks: From Tools to Applications (Oxford University Press, Oxford, 2011).

[19] G. Krings, M. Karsai, S. Bernhardsson, V. D. Blondel, and J. Saramäki, EPJ Data Sci. 1, 4 (2012).

[20] D. R. Cox, Renewal Theory (Methuen, London, 1967).

[21] J. H. P. Schulz, E. Barkai, and R. Metzler, Phys. Rev. X 4, 011028 (2014).

[22] H. Zhu, X. Wang, and J.-Y. Zhu, Phys. Rev. E 68, 056121 (2003).

[23] M. Medo, G. Cimini, and S. Gualdi, Phys. Rev. Lett. 107, 238701 (2011).

[24] J. P. Bagrow and D. Brockmann, Phys. Rev. X 3, 021016 (2013).

[25] M. Boguñá and R. Pastor-Satorras, Phys. Rev. E 68, 036112 (2003).

[26] American Physical Society, APS Data Sets for Research, http://journals.aps.org/datasets.

[27] C. L. Vestergaard, M. Génois, and A. Barrat, Phys. Rev. E 90, 042805 (2014).

[28] H. S. Wilf, Generatingfunctionology (A. K. Peters, Ltd., Natick, MA, USA 2006).

[29] C. Godrèche and J. Luck, J. Stat. Phys. 104, 489 (2001). 